\title{
Principle and kinematic model analysis of a new type of crane telescopic sleeve for marine crane
}

\author{
Guangdong $\operatorname{Han}^{1, a^{*}}$, Hang $\mathrm{Li}^{1, \mathrm{~b}}$, Haiquan Chen ${ }^{1, \mathrm{c}}$, Yuqing Sun ${ }^{1, \mathrm{~d}}$, Jinnan Zhang ${ }^{1, \mathrm{e}}$, \\ Shanghai Wang ${ }^{1, f}$
}

${ }^{1}$ Marine Engineering College, Dalian Maritime University, Dalian China

a18842602951@163.com

\begin{abstract}
Key words: Offshore crane; Telescopic sleeve stabilizer; Principle; Kinetic modeling analysis
Abstract: For offshore crane in the operation process, due to the suspension of lifting wire rope belongs to the flexible parts and wind, wave and ocean environment .These cause the load swing .A new type of offshore crane telescopic sleeve stabilizer was proposed .It uses the casing of the rigid constraints to reduce the swing range of the load .The telescopic pipe device was introduced, and principle of modeling analysis was carried on.
\end{abstract}

\section{Introduction}

Crane refers to a certain range of vertical lifting and horizontal movement of heavy lifting of the crane, and belongs to the material handling machinery. In general, the hoisting machinery is made up of a hoisting mechanism (which enables the load up and down movement), the operating mechanism (to make the lifting machine movement), the variable amplitude mechanism and the rotary mechanism (to move the load). Together with the metal body, power plant, control and necessary auxiliary device combination. The crane is characterized by intermittent and cyclic movements. A working cycle includes: Extract device from the load to the load, and then moved to the designated place to drop down the load, and then carry out reverse motion, so that the device to return to the original place, in order to carry out the next cycle. Generally speaking, crane through the wire rope and hook hanging load, by lifting mechanism for lifting, luffing mechanism and slewing mechanism of horizontal movement. Due to the flexible parts of wire rope, luffing, rotary motion, load will make a simple pendulum movement, which will increase task difficulty and the work cycle time. Especially for ship with cranes, due to the influence of marine environment factors, such as wind, wave and current, the ships have horizontal shake, pitching and sway, sway, surge, heave motion in six degrees of freedom, which has been exacerbated by the load swing, bad sea conditions almost impossible to work.

The mechanical anti-swing and intelligent anti-shake method are usually used for the crane lifting. Mechanical method is used to the crane with Maryland Rigging System [1].The linear dynamic model of the ship crane is established by using Lagrange method, and application of model predictive control [2]. Optimal control method [1] realize the effective control of the swaying of the

Special funds for basic scientific research business of the Central University (132014332,3132015025); Liaoning Natural Science Foundation of China(2015020132)

crane. PARKER and others [3] use RBTS （Rider Block Tagline System） to control the swing of 
load. RBTS belongs to mechanical method. Its construction and control method is easier compared with Maryland Rigging System. But its function is limited when the rope is longer. Yang Wang [4] analysis the swaying under the crane sling for four rope system and pour horoscope rope anti-swing movement characteristics, and puts forward the anti-rolling device of a rigid link.

In intelligent anti-shake, the Sandia national laboratory uses the POS/MV320 TSS navigation system to measure the position of the ship. The inertial measurement unit IMU, gyroscope and GPS are used to measure the movement of the ship [5].The dynamic model of the container crane is established by JANG [6], and the T-S fuzzy control method is adopted to control the swing of the crane. Ismail [7] establishes a three degree freedom model of the ship's rotary arm crane is established. The two order sliding mode control is used to realize the trajectory tracking and swing suppression of the boom, and the impact of the structure is reduced. Pengcheng Wang [8] modeling of the rotary swing arm crane and dynamic analysis. Yongchun Fang [9] based on this model, we designed the nonlinear controller by Lyapunov method, the stability of the closed-loop control system is proved, and the simulation and experimental verification.

Combining with the technology status at home and abroad and the actual situation in a navigation mark ship is proposed in this paper a telescopic mechanical anti-swing device, this paper introduces the principle of crane telescopic tube device for ship, and modeling and analysis.

\section{Structure of telescopic casing device}

The telescopic casing device has a universal torque structure and a telescopic sleeve. The universal torque structure is fixed on the top of the crane, and the bolt rotates, the telescopic casing and the top of the universal torque structure are connected through the screw. The inner tube and the outer tube of the telescopic sleeve are connected with the strengthening plate. The overall structure of the telescopic casing device is shown in Figure 1.The upper half part is the universal torque mechanism, the lower part is the telescopic casing. The upper part of the universal torque mechanism is composed of a hanging ear, a tube body, a damper, a ball hinge. The half part of the telescopic sleeve is made up of the outer tube, the inner pipe and the end cover. The two part is connected with the strengthening board. The retractable wire rope crane can be telescopic tube drive.

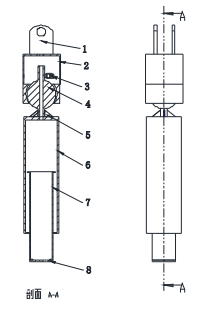

Figure. 1 The overall structure of the telescopic casing device

1.Lug;2.Cylinder;3.Damper;4.Ball hinge;5.Connecting plate;6.Telescopic outer pipe;

7. Telescopic inner tube; 8 . End cap

The universal torque mechanism and the telescopic sleeve are different. The universal torque mechanism is shown in Figure 2.Its anti-shake principle is absorbing the shake by the three damper, damper end is fixed on the inner wall of the cylinder, and the other end of the connecting rod is connected on the ball hinge. When the ship is in motion, the weight of the anti-swing device will be subjected to wave loads, which can lead to the swinging of the telescopic casing, and the telescopic casing is caused by the swinging of the ball joint. Through the relationship between the force and the reaction force, the damper will act on the ball hinge. Due to the effect of damping, the kinetic energy is consumed, and the torque of the telescopic casing is offset by the ball joint, and the load is reduced, and the swing of the telescopic casing is weakened. So through the universal torque 
mechanism can prevent telescopic sleeve and the hanging arm directly and rigidly connected to the hanging ear by large transverse wave loads is pulled off, also can greatly weaken the oscillation of the telescopic pipe, anti-shake effect.

The expansion sleeve is the role of reducing the wire rope. The steel wire rope is passed through the whole device, and the end is connected with the crane by the end cap. The wire rope drives the weight by retracting the lifting and falling. When the weight rises, it will resist the tube in the tube. When the weight drops, the inner tube is due to the weight. In this way, the wire rope is reduced to a great extent, which can solve the problem of heavy load caused by the flexible body.
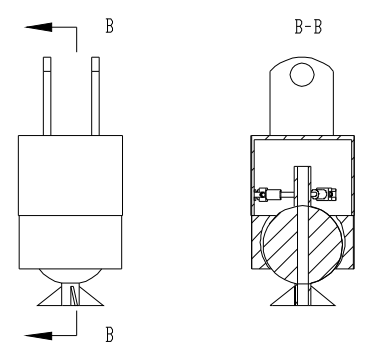

Figure. 2 The universal torque mechanism

\section{Anti-rolling principle of telescopic casing device}

The basic process of the ship crane for deep water: (1) After landing on Mount hook load, structure, variable structure of collaborative action will load from the deck crane. (2)The rotary mechanism, the main arm, the arm of the arm are coordinated to move the load to the surface of the water, adjust the main arm and the arm to ensure that the load is less than the hull;(3)Landing mechanism to load the load to specify the depth of the water. The difference between the operation of the sea and the land on the land is the step (3).Due to the influence of wind, wave and other marine environment, the ship will produce the movement of the roll and pitch, which can increase the load of the crane. So, this paper is focus on the anti-swaying of step (3).

The anti-rolling principle of the telescopic casing device is shown in Figure 3. When the crane boom is raised, the telescopic adjustment of the hydraulic cylinder, the top of the cylinder body around the fulcrum (hanging ears and lifting the top end of the hinge), so that the cylinder is always in a vertical downward state. When lifting heavy loads, because of the wave caused by the ship's rolling and pitching, resulting in a crane to produce a rolling and pitching, so that the load swing. At this point, because the wire rope through the telescopic casing device, so that the universal joint torque mechanism connected to the telescopic casing will withstand a certain wave load, and passed to the joint of the ball joint. At this point, the joint action of the three dampers connected with the ball hinge is offset by the torque of the telescopic casing passing through the ball joint, and the load is reduced. So as to achieve the purpose of anti-roll.

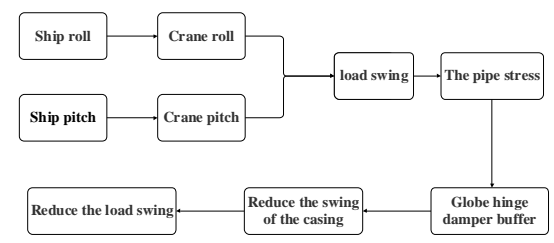

Figure. 3 The anti-rolling principle of the telescopic casing device

\section{Kinematic modeling}

In order to achieve the purpose of reducing the roll of the telescopic casing, we first establish the 
kinematic model of the telescopic casing, and use the Denavit-Hartenberg method to model the system .Three kinematic models are established, respectively. The model of the ship crane, the hanging point and the swing model and the balance torque model of the telescopic casing.

\section{Ship crane point model}

Ship crane lifting diagram model as shown in Figure 4.The corresponding connecting rod parameters are shown in Table 1. Where $\{\mathrm{B}\}$ is the ship coordinate system, $\mathrm{zB}$ is in vertical direction, $\mathrm{xB}$ is beam direction $\mathrm{yB}$ is direction, $\{0\}$ for crane base coordinate system, $\{1\},\{2\}$ are corresponding to the crane rotary joint, variable amplitude joint, $\boldsymbol{\theta}_{1}$ and $\boldsymbol{\theta}_{2}$ respects the two joints, The origin of the $\{2\}$ corresponds to the crane hanging point, L1 is the height of basic,L2 is the length of crane arm.

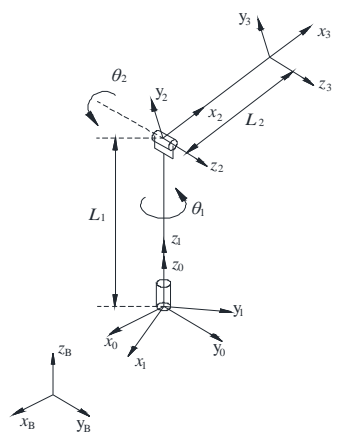

Figure. 4 Ship crane lifting diagram model

Table .1 connecting rod parameters

\begin{tabular}{ccccc}
\hline $\mathrm{i}$ & $\alpha_{\mathrm{i}-1}$ & $\mathrm{a}_{\mathrm{i}-1}$ & $\mathrm{~d}_{\mathrm{i}}$ & $\vartheta_{\mathrm{i}}$ \\
\hline 1 & 0 & 0 & 0 & $\vartheta_{1}$ \\
2 & -90 & 0 & $\mathrm{~L}_{1}$ & $\vartheta_{2}$ \\
3 & 0 & $\mathrm{~L}_{2}$ & 0 & 0 \\
\hline
\end{tabular}

Coordinate $\{i\}$ relative to the coordinate system transform for $\{i-1\}$.

$$
{ }_{i}^{i-1} T=\left[\begin{array}{cccc}
c \theta_{i} & -s \theta_{i} & 0 & a_{i-1} \\
s \theta_{i} c \alpha_{i-1} & c \theta_{i} c \alpha_{i-1} & -s \alpha_{i-1} & -s \alpha_{i-1} d_{i} \\
s \theta_{i} s \alpha_{i-1} & c \theta_{i} s \alpha_{i-1} & c \alpha_{i-1} & c \alpha_{i-1} d_{i} \\
0 & 0 & 0 & 1
\end{array}\right]
$$

In Eq.1, $\cos \boldsymbol{\theta}_{\mathrm{i}}{ }_{\mathrm{c}} \boldsymbol{\theta}_{\mathrm{i}}$ theta, other similar expressions are used in this kind of shorthand.

The transformation matrix of the $\{3\}$ relative to the coordinate system of $\{0\}$ is:

$$
{ }_{5}^{0} T={ }_{1}^{0} T{ }_{2}^{1} T_{3}^{2} T
$$

The transformation matrix of the $\{0\}$ of the crane base coordinate system relative to the ship coordinate system $\{\mathrm{B}\}$ is: 


$$
{ }_{0}^{B} T=\left[\begin{array}{cccc}
1 & 0 & 0 & B_{x} \\
0 & 1 & 0 & B_{y} \\
0 & 0 & 1 & B_{z} \\
0 & 0 & 0 & 1
\end{array}\right]
$$

Where $(\mathrm{Bx}, \mathrm{By}, \mathrm{Bz})$ for the $\{0\}$ crane base coordinate system $\{0\}$ origin in the coordinates of the ship coordinate system $\{B\}$.

If the ship is located at the static sea level, the inertial coordinate system $\{B\}$ and the ship coordinate system $\{\mathrm{N}\}$. When the wind and waves flow disturbed the ship rolling and pitching, the wave motion. The transformation matrix of the $\{\mathrm{B}\}$ of the coordinate system of the ship is in the matrix of $\{\mathrm{N}\}$ :

$$
{ }_{B}^{N} T=\left[\begin{array}{cccc}
c \alpha c \beta & -s \alpha & c \alpha s \beta & 0 \\
c \beta s \alpha & c \alpha & s \alpha s \beta & 0 \\
-s \beta & 0 & c \beta & 0 \\
0 & 0 & 0 & 1
\end{array}\right]
$$

The $\alpha$ is the $B$ of the ship coordinate system and the $\mathrm{N}$ of the inertial coordinate system, and the $\beta$ is the longitudinal wave angle of the ship coordinate system $\mathrm{B}$ with respect to the inertial coordinate system $\mathrm{N}$.

By Eq.2, Eq.3, Eq.4 the $\{5\}$ of the coordinate system is the transformation matrix of the inertial coordinate system.

$$
{ }_{3}^{\mathrm{N}} T={ }_{B}^{N} T_{0}^{B} T_{3}^{0} T=\left[\begin{array}{cccc}
r_{11} & r_{12} & r_{13} & p_{x} \\
r_{21} & r_{22} & r_{23} & p_{y} \\
r_{31} & r_{32} & r_{33} & p_{z} \\
0 & 0 & 0 & 1
\end{array}\right]
$$

The corresponding parameters in Table 1 to (1) - (5), available:

$$
\begin{aligned}
& P_{x}=B_{x} \mathrm{c} \alpha \mathrm{c} \beta-B_{y} \mathrm{~s} \alpha+B_{z} \mathrm{c} \alpha \mathrm{s} \beta-L_{1}\left(\mathrm{c} \theta_{1} \mathrm{~s} \alpha+\mathrm{c} \alpha \mathrm{c} \beta \mathrm{s} \theta_{1}\right)+L_{2}\left(\mathrm{c} \theta_{2}\left(\mathrm{c} \alpha \mathrm{c} \beta \mathrm{c} \theta_{1}-\mathrm{s} \alpha \mathrm{s} \theta_{1}\right)-\mathrm{c} \alpha \mathrm{s} \beta \mathrm{s} \theta_{2}\right) \\
& P_{y}=B_{y} \mathrm{c} \alpha+B_{x} \mathrm{c} \beta \mathrm{s} \alpha+B_{z} \mathrm{~s} \alpha \mathrm{s} \beta+L_{1}\left(\mathrm{c} \alpha \mathrm{c} \theta_{1}-\mathrm{c} \beta \mathrm{s} \alpha \mathrm{s} \theta_{1}\right)+L_{2}\left(\mathrm{c} \theta_{2}\left(\mathrm{c} \beta \mathrm{c} \theta_{1} \mathrm{~s} \alpha+\mathrm{c} \alpha \mathrm{s} \theta_{1}\right)-\mathrm{s} \alpha \mathrm{s} \beta \mathrm{s} \theta_{2}\right) \\
& P_{z}=B_{z} \mathrm{c} \beta-B_{x} \mathrm{~s} \beta+L_{1} \mathrm{~s} \beta \mathrm{s} \theta_{1}-L_{2}\left(\mathrm{c} \theta_{1} \mathrm{c} \theta_{2} \mathrm{~s} \beta+\mathrm{c} \beta \mathrm{s} \theta_{2}\right)
\end{aligned}
$$

Px, Pz, Py for the hanging point relative to the inertial coordinate system of the $\{N\}$ of the yaw, pitch, heave.

In order to realize anti-shake, anti-sway expression is expressed as

$$
\begin{aligned}
& \theta_{1} \rightarrow \alpha \\
& \theta_{2} \rightarrow \beta
\end{aligned}
$$

\section{Hanging point and swing model}

The model of the hanging point and the swing of the pendulum can be represented by a spherical pendulum model: 


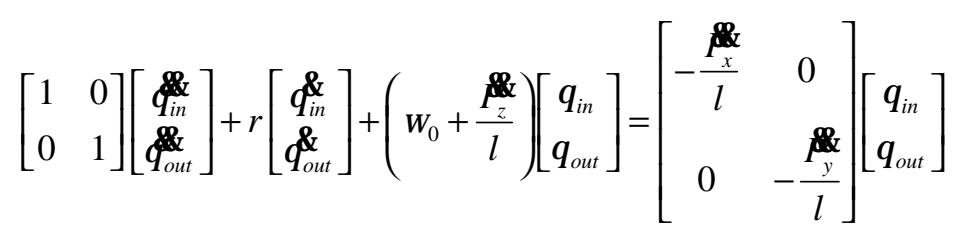

In Eq.9, respectively, the angles, surface corners, $\mathrm{R}$ damping coefficient, the length is 1 ,

$$
\omega_{0}=\sqrt{\frac{l}{g}}
$$

\section{Equilibrium torque model of telescopic casing device}

Moment equilibrium model diagram as shown in Figure 5, Where $\{\mathrm{C}\}$ is the casing coordinate system, Captain $X^{c}$ is the long direction, $Y^{c}$ is the beam direction, $Z^{c}$ is the vertical height.L1 is the rod length between the upper end of the ball joint and the damper joint.L2 is the rod length between the upper end of the ball joint and the end of the telescopic sleeve. F for damper resistance, $\mathrm{G}$ is the force of gravity, F1, F2, F component for G in the vertical direction and casing, the $\alpha$ is the angle between the casing and the $Z^{c}$ axis, the $\beta$ is the angle between the casing and the $X^{c}$ axis.

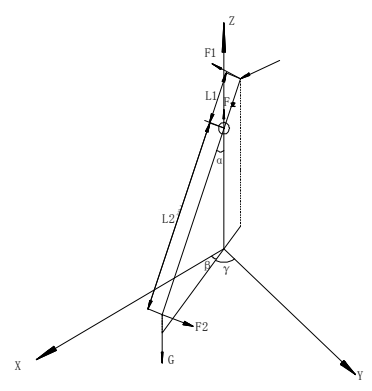

Figure 5 M oment equilibrium model diagram

In order to achieve the torque balance, the principal vector and force of the force system are equal to zero at any point. Expression of analytical expression:

$$
\sum m_{x}(F)=0 ; \sum m_{y}(F)=0 ; \sum m_{z}(F)=0
$$

In order to $\sum m_{x}(F)=0$, so

$$
F_{X 1} L_{X 1}=F_{X 2} L_{X 2}
$$

Because the force $\mathrm{F}$ is provided by the buffer force and the $\mathrm{Z}$ axis is always vertical, and the reaction force of the damper is always opposite to the casing movement direction.so:

$$
F_{X 2}=F \cos ^{2} \alpha \cos ^{2} \beta ; L_{X 2}=L_{2} \cos ^{2} \alpha \cos ^{2} \beta
$$

Because the gravity $\mathrm{G}$ is always downward, and the shear stress of the casing is always perpendicular to the casing.so:

$$
F_{X 1}=G \sin \alpha \cos \alpha \cos \beta ; L_{X 1}=L 1 \sin \alpha \cos \alpha \cos \beta
$$

To sum up can be obtained:

$$
\begin{aligned}
& F L_{2} \cos ^{2} \alpha \cos \beta=G L_{1} \sin \alpha \cos \alpha \cos \beta \\
& F L_{1} \cos \alpha=G L_{2} \sin \alpha
\end{aligned}
$$




$$
\begin{gathered}
F L_{2} \cos ^{2} \alpha \sin \beta=G L_{2} \sin \alpha \\
F L_{1} \cos \alpha=G L_{2} \sin \alpha \\
F_{Z}=G \sin ^{2} \alpha=F
\end{gathered}
$$

In summary, in the ship roll angle $\alpha$, pitch angle $\beta$ conditions, in order to achieve anti-rolling, joint variables, meet Eq.9, the telescopic tube device by force and the buffer to meet Eq.19.

\section{Conclusion}

In view of the special crane operation, this paper presents a new type of marine crane telescopic casing anti-swing device, in the boat with the top of the crane boom, the use of the rigid constraints of the casing and the buffer role of the damper, so that the ship with the crane to maintain a smooth, reduce the load of the swing, so as to reduce the risk of operation. In this paper, the composition and principle of the device is introduced in this paper. Then, the Denavit-Hartenberg method is used to model the system. The model of the ship crane, the hanging point model, the swing model and the balance torque model of the telescopic casing, and finally get the balance torque.

\section{References}

[1] WEN B, HOMAIFAR A, BIKDASH M, KIMIAGHALAM B. Modeling and optimal control of shipboard crane[C]. In Proceedings of IEEE, American control conference, San Diego, CA, June 2-4, 1999: 593-597.

[2] Kimiaghalam B, Homaifar A, Sayrrodsari B. An application of model predictive control for a shipboard crane[C]. Proceedings of the 2001 American Control Conference, Arlington, USA, June, 2001:929-934.

[3] Parker G, Graziano M, Leban F, et. Reducing Crane Payload Swing Using a Rider Block Tagline Control System [J]. OCEANSE, Aberdeen, June, 2007:1-5.

[4] Wang Yang, Design and research of anti-sway device for marine crane Ship science and technology.

[5] Robinett, R. D., Groom, K. N., Feddema, J. T., \& Parker, G. G. Control system and method for payload control in mobile platform cranes [P]. United States Patent. No. US 6,496,765, December $17,2002$.

[6] Jang Jae Hoon, Kwon Sung-Ha, Jeung Eun Tae. Pendulation reduction on ship -mounted container crane via T-S fuzzy model [J]. J. Cent. South Univ., 2012 (19):163-167.

[7] R. M. T. Raja Ismail, Q. P. HaTrajectory. Tracking and Anti-sway Control of Three-dimensional Offshore Boom Cranes Using Second-order Sliding Modes[C]. IEEE International Conference on Automation Science and Engineering (CASE), Madison, WI, 17-20 Aug, 2013:996-1001.

[8] Yongchun Fang, Pengcheng Wang, et. Dynamic analysis and modeling of rotary swing arm crane [J] Journal of Mechanical Engineering, 2011, 47 (20):34-40.

[9] Yongchun Fang, Pengcheng Wang, Ning Sun, et al. Dynamics Analysis and Nonlinear Control of an Offshore Boom Crane [J]. IEEE Transactions on Industrial Electronics, 2014, 61(1):414-427. 